\title{
Enabling Bi-directional Haptic Control in Next Generation Communication Systems: Research, Standards, and Vision
}

\author{
Chathura Sarathchandra, Kay Haensge, Sebastian Robitzsch, Mona Ghassemian, Ulises Olvera-Hernandez
}

\begin{abstract}
Human sensing information such as audio (hearing) and visual (sight) or a combination thereof audiovisual are transferred over communication networks. Yet interacting sense of touch (haptic) and particularly the kinaesthetic (muscular movement) component has much stricter end-to-end latency communication requirements between tactile ends. The statements in this paper, to enable bi-directional haptic control, indeed follow the widely accepted understanding that edge computing is a key driver behind Tactile Internet aiming to bring control and user plane services closer to where they are needed. However, with an updated wider analysis of (pre)standardisation activities that are chartered around Tactile Internet, this paper highlights the technology gaps and recommends open research topics in this area.
\end{abstract}

Index Terms-Tactile Internet, B5G, Haptic, Ultra-low latency communication, Use Cases, IEEE, 3GPP, IETF, Standards

\section{INTRODUCTION}

$\mathbf{H}$ APTIC information in immersive communication enables mediated touch (kinaesthetic and/or tactile cues) over a computer/communication network to feel the presence of a remote user and to perform social interactions including handshake, pat, or hug. The application spectrum for haptic technology extends from social networking, gaming and entertainment to industry operation, training, and health care. The bi-directional haptic control system comprises a local master user, a remote controlled user, a remote user model at the local domain, and a local user model at the remote domain which can be split into three distinct domains as shown in as depicted in Fig. 1]: the local master domain (including the operator and the haptic command interface), the tactile network domain (providing the medium for bilateral real feel communication between master and controlled domains), and the remote controlled domain (teleoperator and the haptic feedback interface). The human models (remote participant or local user) can be either a physical entity (such as a social robot) or a virtual representation (such as a virtual reality avatar). Maintaining a human model for remote use involves the exchange of haptic data (position, velocity, interaction forces, etc.) and non-haptic data (gestures, head movements and posture, eye contact, facial

Chathura Sarathchandra, Sebastian Robitzsch, Mona Ghassemian are with InterDigital Europe, Ltd.

Kay Haensge is with InterDigital Germany, $\mathrm{GmbH}$

Ulises Olvera-Hernandez is with InterDigital Canada, Ltée,

E-mail: \{firstname.lastname\} @interdigital.com expressions, user's emotion etc.). Typically, haptic information is composed of two distinct types of feedback: kinaesthetic feedback (providing information of force, torque, position, velocity, etc.) and tactile feedback (providing information of surface texture, friction, etc.). The former is perceived by the muscles, joints, and tendons of the body. The tactile feedback should not be confused with the Tactile Internet (TI) [1] whereas the latter is consumed by the mechanoreceptors of the human skin. While the exchange of kinaesthetic information closes a global control loop with stringent latency constraints, this is typically not the case with the delivery of tactile impressions. In case of non-haptic control, the feedback is audio/visual and there is no notion of a closed control loop. In addition to enabling haptic/non-haptic control/data, the TI aims to enable an overlay low latency network platform, providing interoperability over different communication technologies, e.g., over 5G Ultra-Reliable Low-Latency Communication (URLLC), Time Sensitive Networking (TSN), etc.

The main contributions of this paper are in two folds: a) we provide a list of relevant standardisation activities addressing different aspects of bi-directional haptic communication and control, b) we highlight the technology gaps and open research topics to reach to the vision for realisation of the Tactile Internet. The rest of the paper is organised as following: Section III discusses the TI resource sensitive use-cases and requirements and presents early adaptors of the technology. Section III presents related standards and bodies addressing different aspects of the TI technology mainly on the architecture as well as data level. Existing technology enablers for a low latency communication platform are described in Section IV as required for the haptic data mode to be transmitted or perceived in immersive applications. Section V highlights research and innovation opportunities to address the technology gaps to overcome the limitations as the result of distance projected by the speed of light when transmitting sense of touch over a distance. Finally, Section VI concludes the paper and presents recommendations to the technology providers and standard organisations.

\section{Resource-Sensitive Use-CASes And REQUIREMENTS}

The TI will enrich the conventional audio/visual transport by including the ability of haptic control and provide the medium for transporting touch and actuation in real-time. The TI has a wide spectrum of use cases and applications ranging from 


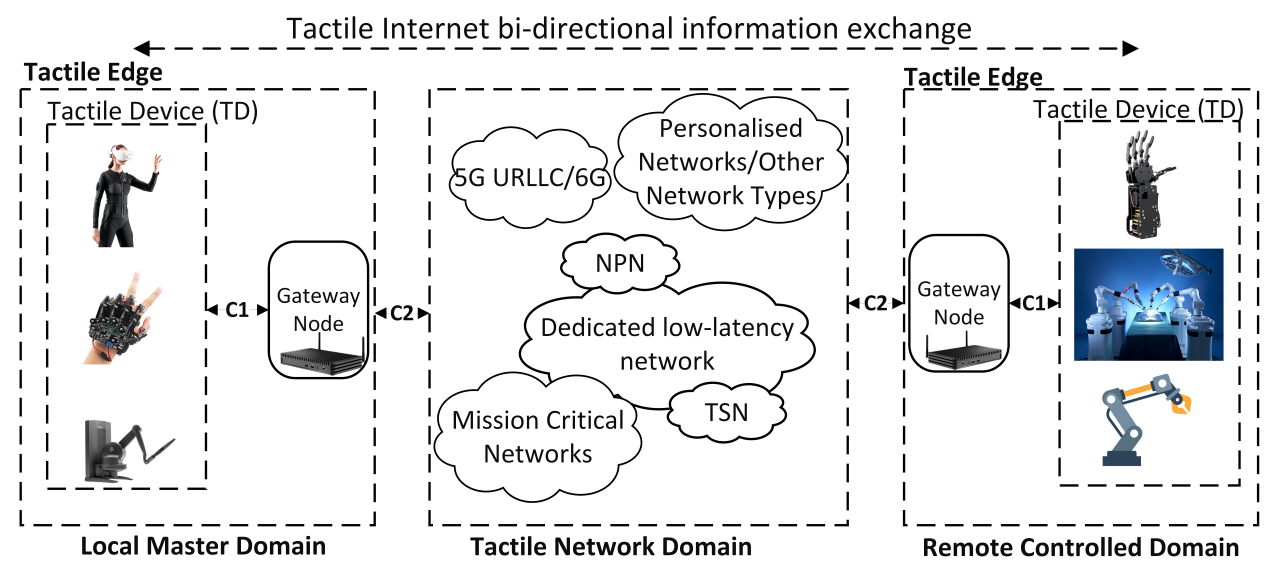

C1: Intra-network connectivity in master or controlled domain

C2: First/last mile connectivity to master or controlled domain

Fig. 1. Overview of Bi-directional Haptic Control [1] [2]

use cases requiring URLLC to ones with infrequent sampling of haptic data over less stringent networking modes.

In this paper, we focus on resource sensitive use cases, and define the operational states and technical requirements in which a Tactile Device (TD) should exist, over the course of its operation. Several TI use cases are described and for each use case scenario, technical challenges, and advancements that TI platform can provide are discussed. The use cases are surgical robotics (Tele-surgery), remote expert assistance in repairs (Smart factory) and multi-player interactive gaming and for Virtual Reality (VR)/Augmented Reality (AR) interaction, and human communications (Tele-presence).

The main application requirements for delivering a bidirectional closed haptic communication and control by the network are ultra-low latency, high availability, reliability, and security to enable the haptic data and feedback interactions. From a large range of applications discussed in literature, based on conducted interviews with different verticals, the early adopters of haptic technology are expected to be in the order of following:

- New generation of entertainment: Multiplayer games and entertainments where haptic wearables with sensory replacement, sensory augmentation enrich the interaction between the players in different locations,

- Cooperative manufacturing: Remote operation/mentoring of engineers to work from a safe distance to the hostile environment where multisensory (including Extended Reality (XR) and haptic technologies) is required to enable the remote expert to guide at the precise location of the operation to the present worker in the factory,

- Interactive tele-care /tele-surgery: Tele-surgery and telementoring for remote operation in rural areas where security, high reliability and extremely low latency communication is required to interact with haptic data/feedback.

The latency (end-to-end round-trip time) requirement is suggested to be in the milliseconds which represents the human reaction time. Yet for industry and robotic applications, the sensitivity of control circuits when controlling devices moving rapidly can require an end-to-end latency in submillisecond [3] per sensor/actuator.

\section{RELATED WORKS AND STANDARDS}

This section provides an overview of standardisation bodies that explicitly work on TI specifications or directly relate to TI through architecture, use cases, focus groups, or study items.

\section{A. IEEE}

The IEEE TI Standards Working Group (WG), designated the numbering IEEE 1918.1, undertakes pioneering work on the development of standards for the TI as defined by the International Telecommunication Union (ITU) in August 2014. The IEEE TI working groups initiated in 2016 [1] aim to develop baseline standard addressing the network architecture as well as haptic codecs for the TI providing a fast, reliable, secure, and available platform as part of the service requirements of the $5 \mathrm{G}$ and beyond.

The TI haptic codec standardisation WG provides codecs for the TI enabling the interoperability of different haptic (kinaesthetic and tactile) input and output devices as required to achieve necessary market scale in the realisation of TI technologies, devices, and applications. Haptic codecs for the TI address TI application scenarios with human in the loop (e.g., teleoperation/remote touch applications) as well as machine remote control which define (perceptual) data reduction algorithms and schemes for closed loop (kinaesthetic information exchange) and open loop (tactile information exchange) communication. Furthermore, specific mechanisms and protocols for the exchange of the capabilities with respect to the workspace, the robot arm's number of Degree of Freedom (DoF), haptic signal amplitude range, temporal and spatial resolution of the haptic devices are included in the IEEE TI standard scope. A fundamental challenge in context of the Tactile Internet is the development of a standard haptic codecs family, like the state-of-the-art audio (ITU Telecommunication Standardisation Sector (ITU-T) H.264) and video 
(ISO/IEC MPEG-4) codecs [4]. Embracing both kinaesthetic as well as tactile information, such a codec family would be a key enabler for scalability at the network edge and universal uptake. Furthermore, it introduces a layered approach to haptic data (comprising multi-modal sensory information), which would be crucial for operation in typically challenging wireless environments.

Use case scenarios and requirements, technical assumptions, definitions, elements, functions, interfaces, and other related consideration are scoped in the architecture WG. TI architecture also includes the novel aspects and differentiating factors compared with, e.g., 5G URLLC where it is noted that the $\mathrm{TI}$ and associated requirements that the standard must serve to be likely operated as an overlay on other networks or combinations of networks.

\section{B. IETF}

Tactile Internet has been discussed in a number of groups within IETF primarily as a use case which demands for improving networking technologies towards satisfying its stringent resource requirements. Activities in Network Function Virtualisation (NFV) Research Group (NFVRG) suggests that a combination of radio access and core network components must be isolated into network slices for addressing specific requirements of emerging use cases, such as TI services [5]. Computing In the Network Research Group (COINRG) [6] highlights requirements on in-network computing for providing real-time interactivity for immersive and mobile applications with tactile and time-sensitive data, which includes (but is not limited to), new internet architectures at the edge for improved performance, enabling joint collaboration, higher layer protocol optimisation to reduce latency and, enabling multi-stream, multi-device and multi-destination applications.

A recent submission in DISPATCH working group [7] introduces haptic data as a top-level haptic media type, and the recent acceptance of 'haptics' as a first-order media type in ISO Base Media File Format (ISOBMFF), making it historically the first attempt at registering haptics as a media format. Making haptics a top-level media type allows for the definition of data formats for haptic sub-modalities (e.g., kinaesthetic, vibrotactile) in a more streamlined manner. Given this development the authors make a case for haptic technology to be added to the list of top-level media types recognised by IETF.

\section{3rd Generation Partnership Project (3GPP)}

From the outset, 3GPP 5G System (5GS) were designed to provide highly reliable (e.g., URLLC), service based, low latency communications (e.g., Edge Computing) and enablers for Industrial Automation, e.g., Time Sensitive Communications (TSC), and Network Slicing. Although great progressed was achieved, many existing and new Use Case (UC)s still remain to be addressed, e.g., UCs with stringent requirements, as those needed to support tactile and multi-modal communication services over the $5 \mathrm{G}$ system. To address these challenges, 3GPP TR 22.847 intends on creating a gap analysis between new potential requirements and existing requirements and functionalities supported by 3GPP. Especially,for use cases that are immersive real-time experiences, including closedloop feedback and control under varying DoFs.

Requirements for cases under consideration include (but are not limited to), parallel transmission of multiple modality representations associated with the same application. Also, their reliability, availability, security, privacy, charging, and the identification of Key Performance Indicator (KPI) for specific use cases are considered. 3GPP TR 22.847 provides an example of new requirements to further enhance 3GPP 5GS to meet the needs of demanding applications as those seen in the healthcare industry.

3GPP 5GS concepts, e.g., those explored in 3GPP TR 22.858 address deployments in residential environments, such as homes and small offices, includes both have wired and wireless converge. These concepts help us visualise where these systems may be deployed and where new requirements lay, including both 3GPP and Non-3GPP Accesses. Services deployed on fixed/wired networks differ from those deployed in mobile networks, as mobile devices tend to be addressed individually, while in fixed networks, devices on a LAN are addressed through a gateway, and they are typically not known to the core Network.

In residential/small environments this gateway is referred to as evolved Residential Gateway (eRG) and they are considered from a 3GPP System perspective as User Equipment (UE). Nevertheless, they also provide connectivity and Quality of Service (QoS) handling to other devices connected behind their realm, e.g., Personal IoT networks (PIN), which might correspond to sensors or actuators remotely controlled by e.g., a factory worker or a physician. It is thus quite possible that in an End-to-End scenario where a surgeon wearing a tactile glove, which is a wireless device connected through an eRG, may connect to actuators in a remote location, far away from the surgeon. In that case, the eRG at each end of the connection will need to satisfy reliability and latency constrains, using technologies that may further extend the existing 5GSs

\section{D. $I T U-T$}

The Focus Group on Technologies for Network 2030, which concluded its work in Summer 2020, studied the capabilities of networks for the year 2030 and beyond. The group selected TI as a representative use case for network 2030, among other use cases such as holographic type communications and Spaceterrestrial integrated network [3].

\section{Existing Technology Enablers And Testbeds}

This section presents the technology enablers for the Local Master, Remote Controlled and Tactile Network Domains as depicted in Fig 1. Furthermore, a number of related experimental testbeds are listed with a mapping to the discussed standards.

\section{A. Unified Data Link}

Any device requires a link local networking technology to communicate with another endpoint that has access to the same 
medium independent from its type, e.g., wired, wireless or optical. With the adoption of IEEE 802.3 - widely known as Ethernet - as the data link layer protocol for private and industry Local Area Networks (LANs) and eventually as the link layer protocol that runs the internet, many access technologies for devices followed suit such as WiFi, Bluetooth, Zigbee, LiFi and 3GPP's user plane with the release of 5G. Not only does such a unified access technology enable a rather homogeneous communication environment across a range of devices, it also lowers the complexity of supporting a range of data link layer in operating systems based on their physical layer realisation. More importantly, it allows TI enabling technologies, such as TSN, to work across all three TI administrative domains (as illustrated in Fig. 11) for the delivery of packets in a guaranteed time, which is often referred to as "real time".

\section{B. Advances in Communication Systems}

The Network Domain in Fig. 1 illustrates a range of technology areas that are capable to deliver on the TI requirements, as described in Section II In the cellular telecommunication area, 5G has paved the way to support URLLC use cases on the user plane and has even seen the adoption of cloud concepts in their system architecture aka Service-based Architecture (SBA) in 3GPP TS 23.501, where the realisation of control plane functions follow cloud native concepts ensuring highly available, flexible and reliable services. Additionally, 5G systems come with a strong notion of QoS enforcement procedures on the user plane combined with resource isolation procedures making it a significant technology enabler for interconnecting Tactile Edges (if Tactile Devices (TDs) do not operate in the same Tactile Edge). Furthermore, the concept around Non-public Networks (NPNs) can be seen as another technology enabler for TI use cases where the 5G system is a fine tuned and focused solution for a specific use case without the need to support a wide range and diverse set of applications.

\section{Edge Computing}

Moving services to the cloud has tremendously changed the landscape of service provisioning, reliability, and scalability. However, KPIs of sub-5ms latency and $1 \mathrm{Gbps}$ per user only allowed one logical move for services: if the network between client and servers cannot deliver those KPIs the service must come closer to the user. Combined with the advances of NFV and private cloud solutions, edge computing is a key enabler in the context of TI and offers a programmable and homogeneous framework to manage compute and virtual instances across these compute nodes. With the service orchestration frameworks that manage the lifecycle states of service instances across the edges, edge computing is a key technology enabler for achieving KPIs important for TI services by bringing the service closer to the TD or offering processing or storage intense service functionality to the edge.

\section{Terminals}

In the local master domain various sensors, actuators, display devices are used to provide a realistic haptic interaction with the remote devices in the controlled domain. The sensor components capture the tele-manipulation instructions (e.g., kinaesthetic) in the master domain, and the resulting changes in the control domain (e.g., haptic feedback) are shown in Figure 1. Actuators, in both local master and remote control domains, execute the user's tele-manipulation instructions. The number of independent coordinates used for providing the end user experience at the master domain (using Human System Interfaces), and for controlling the velocity, position, and the orientation of the controlled devices is defined by their DoF. Today, there exist devices with different DoF capabilities and are used match varying requirements of use cases [1]. Advanced perceptual coding as well as resource management/task offloading techniques may be used for optimising communication and computing resources.

\section{E. Testbeds, Demonstrations and Trials}

This section presents existing TI testbeds, categorising ones that relate to TI specific standards (IEEE 1918.1) in IV-E1 and ones that do not in IV-E2

1) Standards Oriented Testbeds: An Extensible Testbed for TI Communication (TIXT) [2] implements a generic TI architecture which is in line with the TI architecture proposed in IEEE 1918.1 [1] standards working group on TI. By implementing a standards compliant testing environment it attempts to create a common ground for testing and evaluating TI research performed by various groups. TIXT's implementation is highly extensible with its loosely coupled components using standard communication interfaces. TI scenarios with a combination of simulators/emulators and real hardware can easily be incorporated into the same experiment.

The Haptic Communication Testbed at the Otto-vonGuericke university of Magdeburg (OVGU-HC) [8] provides a data-driven experimentation platform. OVGU-HC has been designed targeting TI experiments over small wireless networks as well as long-distance teleoperation scenarios over the internet. The platform provides a means for automating experiment scheduling and deployment through a generalised experiment description language. The authors demonstrate that the proposed platform is capable of implementing protocols and codecs presented in IEEE 1918.1 standards.

2) Non-Standards Oriented Testbeds: A design approach has been presented [9] for provisioning TI services in Virtual Network Function (VNF)-based Multi-access Edge Computing (MEC) systems. The authors develop, implement, and evaluate CALVIN, a low-latency management framework for distributed Service Function Chaining (SFC). A primary design choice aims at reducing computing latency by eliminating the context switching overhead due to shared virtual CPU between the kernel and user space, by implementing VNFs either purely in the kernel or user space, instead of utilising both together. Experimental results performed in a real testbed indicate that CALVIN can significantly reduce latency.

A Software-defined Networking (SDN)- and NFV-enabled experimental platform for $5 \mathrm{G}$ has been proposed for supporting TI industrial applications [10]. The testbed consists of three main layers (Backend, Field, and Network). The backend layer includes OpenStack based cloud resources where 
cloud applications are deployed. The network layer consists of SDN/NVF based industrial networks. The Network layer interconnects the compute nodes with the Field layer via virtualised Industrial Internet of Things (IIoT) gateways and provides network slicing to support multiple applications. The virtualised IIoT gateway interconnects smart sensors and smart light actuators with the backend cloud. It also enables to migrate VNFs down to the field, allowing for services with ultra-low latency requirements to be migrated to the edge for minimising latency.

TCPSbed [11] presents a design of a testbed for Tactile Cyber Physical Systems (TCPS). Specifically, TCPS incorporates sensory feedback into CPS. Since, controlling latency and ensuring stability is crucial for TCPS applications, it allows isolation of latency of individual components of live applications, fine-grained characterisation of latency and control performance. Moreover, TCPSbed allows for both real and emulated networks and applications that mimic real-world embedded components for better emulating varying realistic conditions.

\section{RESEARCH AND INNOVATION OPPORTUNITIES}

There are a number of potential solutions for interacting haptic data within the latency constraints. In this section, we provide open areas for research and innovation from data, network, and terminal levels.

\section{A. Haptic Data Transmission and Perception}

There are a number of advanced techniques that can be exploited to support haptic data transmission and perception:

Artificial Intelligence (AI) and Machine Learning (ML) techniques allow creating a perception of the sense of touch within intelligent edge solutions for movement/haptic prediction to reduce latency. The multimodality of the situation can further support predicting the haptic information at the remote controlled domain either by learning the movement models or predicting them using audio/visual information to reduce the perceived latency.

Secure and reliable transport solutions differ for haptic data. Various low overhead transport protocols are proposed and implemented by research communities with a few being standardised by IEEE, 3GPP, and IETF standard organisations or developed as a proprietary solution. Considering TI as an overlay network that can run different transport layer protocols and solutions in each part of the network, this can lead to interoperability issues and adding to the e2e delay.

Haptic data has been recently been accepted as a first-order media type in popular media formats such as MPEG ISO Base Media File Format [7]. Efficient streaming techniques for transmitting haptic data along with any combination of existing (e.g., audio/visual) and new media types (e.g., ambience, emotion) must be further investigated (e.g., audio/haptic, audio/visual/haptic, audio/visual/haptic/emotion). Haptic submodality (e.g., kinaesthetic, touch) aware networking is another advanced open research topic. Haptic feedback can be different based on the application that it is being used for and the user interacting with the environment and the tactile device.
Dynamic slice/edge relocation during a session based on user haptic experience feedback (e.g., telepresence of a family meeting may only use haptic feedback to communication emotion) or haptic interaction prediction based on other data modes (e.g. hug/handshake at the start and end) can improve the network resource utilisation. Additional signalling and functionalities are required to enable the utilisation of higher cost slice/edge resources only when needed during a session.

\section{B. Service-centric System Architecture}

The adoption of cloud native principles in 5G systems has a much greater impact on the overarching system design than simply turning monolithic network functions into microservices and orchestrate them into islands of microservice management systems inside the TI network or Tactile Edge domain. One of the key design choices when realising an application in a cloud native fashion is the separation of routing, monitoring, analytics, and orchestration from the actual application whose main objective is to focus on processing incoming requests and returning a response accordingly. The naming ontology of services over the internet, i.e. Fully Qualified Domain Names (FQDNs), is being used to allow the logical separation of functions which form the application. Thus, each client (5G Core (5GC) consumer, UE, TD, gateway node or client process of a vertical application) is fully aware about the FQDN of the next function (server) which can serve the request the client aims to issue.

However, when distributing control or user plane (micro)services across a range of compute hosts (inside the Tactile Edge (TE) or inside the Tactile Network domain) and expecting to freely change their lifecycle or even orchestration state, challenges around dynamic and scalable name resolution and packet routing must be addressed through standardisation and adoption.

As the TE can be equipped with a dedicated gateway node, as illustrated in Fig. 1. the network domain will see the gateway as the UE. This results a hard boundary between TDs and the Tactile Network Domain which especially when it comes to network attachment, mobility and user authentication procedures (e.g., the Non-Access Stratum (NAS) protocol in $5 \mathrm{G})$. For the realisation of a service-centric system architecture that sees everything as a service operating on top of a connectivity and routing layer, an evolution of NAS and the likes are required to decouple their functionality from a static binding to a hardware component.

\section{Terminal Innovation}

New sensory and haptic functionalities required by applications of TI further increases the demand on processing power required to execute the computation-intensive and low-latency tasks, while meeting stringent requirements of TI. However, terminal/tactile devices (TD) today have limited computing resources, and local processing of such tasks may not meet the latency requirements of TI. Moreover, devices used by many TI applications (e.g., robots, drones) contain only a limited supply of power. 
Offloading computation-intensive tasks from such resource scarce devices to be executed distributedly across resources over various networks (Fog, Edge, Cloud), towards minimising the response time and power consumption has recently gained popularity and has been identified as a crucial technique for improving TI applications [12]. TI computation offloading frameworks must provide efficient function partitioning and offloading methods considering new device types (see section IV-D of TI applications. Offloading algorithms must aim for minimising the total time spent on offloading. Moreover, improved resource allocation and management of resources at each constituent resource providers (e.g., Radio, Non-Radio, Computing) in the end-to-end system is paramount to reducing the total response time when offloading.

The incorporation of various human-system interfaces (TD in Figure 1) in TI (with specific hardware functionalities) and the distributed execution of functions are key aspects of terminal evolution in TI. The dynamic inter-connection of these distributed functions and TDs can be seen as the composition of various hardware and software functionalities towards providing/improving the user's experience. The haptic and bidirectional communication brings consumption of experiences closer to humans (as opposed to experiences that are consumed today through mobile devices, for example).

This increasingly distributed and human-centric experiences that are enabled by TI require new and improved means for gathering and enforcing QoS and Quality of Experience (QoE) metrics. Such requirements must be enforced throughout the distributed system, for improving not only the latency and reliability, but also the user's haptic, visual, and audio experiences. However, for ensuring requirements in more dynamic scenarios where systems must adapt to satisfy/maintain requirements, dynamic methods for capturing both objective and subjective metrics of user experiences must be provided.

\section{CONCLUSION}

Advancements in haptic technology, including high levels of co-presence in real time, which demand ultra-reliable low latency platforms over distance, offer high quality interpersonal communication experiences remotely. In this paper, we have provided an overview of the TI research and developments in view of addressing further advancements required in the domain for realisation of the bi-directional haptic control capabilities in relevant industrial, entertainment and healthcare use cases. We have presented the related standardisation activities for haptic communication mainly by 3GPP, IEEE and IETF. A number of existing testbeds are discussed and categorised according to the standards used in the implementation. Considering the existing technology enablers, we highlight a number of open areas of research that a) are required for the interoperability between the discussed standards in order to realise a communication platform within millisecond and submilliseconds latency depending on the use case requirements, b) highlight advancements in other technologies (e.g., AI/ML) that can support improvement in haptic control scenarios, c) present terminal innovations that can evolve into immersive communications, including bi-directional haptic control.
While there are a number of activities in the standardisation organisations, TI is not in the forefront in many Standard Development Organisation (SDO) groups and is exploited mostly as a use case. Further consistency and coordination across the various groups are required to establish and bring the various efforts by SDOs under one umbrella.

\section{REFERENCES}

[1] O. Holland, E. Steinbach, R. V. Prasad, Q. Liu, Z. Dawy, A. Aijaz, N. Pappas, K. Chandra, V. S. Rao, S. Oteafy, M. Eid, M. Luden, A. Bhardwaj, X. Liu, J. Sachs, and J. Araújo, "The ieee 1918.1 "tactile internet" standards working group and its standards," Proceedings of the IEEE, vol. 107, no. 2, pp. 256-279, 2019.

[2] V. Gokhale, K. Kroep, V. S. Rao, J. Verburg, and R. Yechangunja, "Tixt: An extensible testbed for tactile internet communication," IEEE Internet of Things Magazine, vol. 3, no. 1, pp. 32-37, 2020.

[3] ITU, "Representative use cases and key network requirements for network 2030," International Telecommunication Union, Technical Report (TR), January 2020, focus Group - Network 2030.

[4] A. Aijaz, M. Dohler, A. H. Aghvami, V. Friderikos, and M. Frodigh, "Realizing the tactile internet: Haptic communications over next generation 5g cellular networks," IEEE Wireless Communications, vol. 24, no. 2, pp. 82-89, 2017.

[5] C. J. Bernardos, A. Rahman, J. Zuniga, L. Contreras, P. Aranda, and P. Lynch, "Network virtualization research challenges," IRTF draft draftirtf-nfvrg-gaps-network-virtualization-10, 2019.

[6] I. Kunze, K. Wehrle, D. Trossen, and M. Montpetit, "Use cases for in-network computing," IRTF draft-irtf-coinrg-use-cases-00, 2021.

[7] Y. K. Muthusamy and C. Ullrich, "The 'haptics' top-level media type," IRTF draft-muthusamy-dispatch-haptics-01, 2021.

[8] F. Engelhardt, J. Behrens, and M. Güneş, "The ovgu haptic communication testbed (ovgu-hc)," in 2020 IEEE 31st Annual International Symposium on Personal, Indoor and Mobile Radio Communications, 2020, pp. 1-6.

[9] Z. Xiang, F. Gabriel, E. Urbano, G. T. Nguyen, M. Reisslein, and F. H. P. Fitzek, "Reducing latency in virtual machines: Enabling tactile internet for human-machine co-working," IEEE Journal on Selected Areas in Communications, vol. 37, no. 5, pp. 1098-1116, 2019.

[10] P. Mekikis, K. Ramantas, A. Antonopoulos, E. Kartsakli, L. SanabriaRusso, J. Serra, D. Pubill, and C. Verikoukis, "Nfv-enabled experimental platform for $5 \mathrm{~g}$ tactile internet support in industrial environments," IEEE Transactions on Industrial Informatics, vol. 16, no. 3, pp. 1895-1903, 2020.

[11] K. Polachan, T. V. Prabhakar, C. Singh, and F. A. Kuipers, "Towards an open testbed for tactile cyber physical systems," in 2019 11th International Conference on Communication Systems Networks (COMSNETS), 2019, pp. 375-382.

[12] N. Promwongsa, A. Ebrahimzadeh, D. Naboulsi, S. Kianpisheh, F. Belqasmi, R. Glitho, N. Crespi, and O. Alfandi, "A comprehensive survey of the tactile internet: State-of-the-art and research directions," IEEE Communications Surveys Tutorials, vol. 23, no. 1, pp. 472-523, 2021.

Chathura Sarathchandra is a researcher at InterDigital. He received his B.Sc. Hons. and Ph.D from the school of Computer Science \& Electronic Engineering, University of Essex, U.K., and he was awarded the UEssex scholarship for pursuing his Ph.D. Chathura has worked on various private, national, E.U. and international projects. He has held several IEEE \& ACM program committee memberships (e.g., ACM SIGCOMM, IEEE CloudCom, IFIP Networking). His current research interests fall within the general area of future computing technologies, including virtualization and mobile computing. 
Kay Haensge is a researcher at InterDigital Germany GmbH. He studied Information and Communication Technology at University of Applied Sciences in Leipzig and wrote his master's thesis about QoS-based WebRTC Service Delivery over a Next Generation Mobile Network (NGMN) Infrastructure He worked as a researcher at the Deutsche Telekom AG to support the standardization group within the 3GPP\#SA2 TSG. After joining InterDigital, he continued working within EU-funded projects as well as support of standardisation activities within the scope of system architecture of $5 \mathrm{G}$ network elements.

Sebastian Robitzsch is a Senior Staff Engineer at InterDigital and works on beyond $5 \mathrm{G}$ architecture innovations in the area of location-aware cloudnative orchestration, programmable systems combined with service routing capabilities. In the past he has been with Dublin City University, T-Systems, Fraunhofer FOKUS, and Nokia Research Centre. Sebastian received his Ph.D. from University College Dublin, Ireland, and an M.Sc. equivalent (Dipl.-Ing. (FH)) from the University of Applied Sciences Merseburg, Germany in 2008.

Mona Ghassemian is a senior R\&I manager at InterDigital. She received her $\mathrm{PhD}$ from King's College London (KCL) and continued at KCL as a research associate in E-Sense (FP6- EU project) for 2 years. She worked as a lecturer and senior lecturer at KCL, Uni of Greenwich and SBU, teaching courses and supervising at $\mathrm{BSc}$, MSc and $\mathrm{PhD}$ levels for 12 years prior to her move to $\mathrm{BT}$ research in 2019. Mona is a senior member of IEEE, the IEEE UK \& Ireland section chair (2020-2021) and a member of IEEE Tactile Internet SA WG.

Ulises Olvera-Hernandez is a Senior Principal Engineer for InterDigital, responsible for developing 5G 3GPP System Architecture Evolution Standards Prior to joining InterDigital, Ulises spend 15 year at Ericsson in Mexico, Canada, Sweden and Ireland developing Cellular Systems for International markets. Ulises holds a bachelor's degree in Mechanical and Electrical Engineering from National Autonomous University of Mexico(UNAM). Ulises has co-authored more than 150 granted patents, and he has more than 30 years of experience in the Research and Development of Cellular Systems, with emphasis in the development of the 3GPP 5G Next Generation Network and beyond. 